\title{
L’activité de la rougeole au Canada : Janvier à juin 2014
}

\author{
Deehan $\mathrm{H}^{1 *}$ et A Shane ${ }^{1}$ \\ 1 Agence de la santé publique du Canada \\ * Auteure-ressource : Heather.Deehan@phac-aspc.gc.ca
}

\begin{abstract}
Résumé
Depuis le début de 2014 et jusqu'au 10 mai, cinq provinces ont signalé 103 cas de rougeole à l'Agence de la santé publique du Canada : la Colombie-Britannique, l'Alberta, la Saskatchewan, le Manitoba et l'Ontario. Trois facteurs contribuent à cette situation. Premièrement, les Canadiens voyagent plus qu'auparavant, ce qui augmente le risque que ceux qui ne sont pas immunisés importent la maladie au Canada. Deuxièmement, on a observé une augmentation de l'incidence de la rougeole dans des pays où les échanges de population avec le Canada sont élevés, notamment la France (2011), les Pays-Bas (2013) et, plus récemment, les Philippines (2014). Enfin, la couverture vaccinale est sous-optimale dans certaines régions de tout le Canada. Cette année, jusqu'à maintenant, on a signalé 21 cas importés rapporté au 10 mai; néanmoins, malgré le fait que la rougeole est très contagieuse, seules huits de ces importations ont donné lieu à des transmissions au Canada. Afin d'éliminer la rougeole de manière durable, il faut nécessairement renforcer les programmes d'immunisation ainsi que continuer à assurer une vigilance accrue et à endiguer rapidement les cas d'infection importés.
\end{abstract}

\section{Introduction}

Malgré la réussite de l'élimination de la rougeole au Canada, on s'attend à ce qu'il y ait des importations de cas de rougeole et une propagation secondaire subséquente, tant que cette maladie reste endémique ailleurs dans le monde. Voici un résumé de l'activité de la rougeole au Canada en 2014.

\section{Résumé épidémiologique}

Depuis le début de l'année 2014 (de la semaine épidémiologique 1 du 30 décembre 2013 à la semaine épidémiologique 19 du 10 mai 2014), on a signalé 103 cas de rougeole à l'Agence de la santé publique du Canada (l'Agence), par l'intermédiaire du Système canadien de surveillance de la rougeole et de la rubéole. Cinq provinces ont signalé des cas : la Colombie-Britannique $(n=36)$, l'Alberta $(n=24)$, la

Saskatchewan $(n=16)$, le Manitoba $(n=8)$ et l'Ontario $(n=19)$. En Colombie-Britannique, la Fraser Health Authority a averti l'Agence et le public à propos d'une importante éclosion dans la région de Fraser East, où une estimation a dénombré 423 cas, dont la plupart n'ont pas encore été signalés par l'intermédiaire du système de surveillance national.

Pour l'instant, on a signalé 21 cas, importés de six pays. La majorité de ces cas importés proviennent des Philippines $(n=15 ; 71 \%)$, où une importante éclosion de rougeole s'est produite, mais des cas ont aussi été importés de l'Inde $(n=2)$, des États-Unis $(n=1)$, de la Thaïlande $(n=1)$, du Pakistan $(n=1)$ et de I'Italie/Amsterdam $(n=1)$. Le nombre de cas de rougeole importés au Canada est demeuré relativement stable au cours des années, avec une médiane de cinq par année, de 1998 à 2009. Cependant, on a récemment observé des hausses, en 2010 ( $n=10$; année des Olympiques), en 2011 ( $n=29)$ et en $2013(n=12)$. Les modifications des habitudes de voyage et de l'incidence mondiale de la rougeole sont deux facteurs contributifs qui peuvent expliquer l'augmentation de ces importations.

On estime qu'en 2000 , les résidents canadiens ont entrepris 4,5 millions de voyages à l'étranger, alors que des visiteurs de l'étranger (non-résidents) ont effectué 4,4 millions de voyages au Canada (1). On estime qu'en 2012, les Canadiens ont effectué 11 millions de voyages à l'extérieur du pays (à l'exclusion des voyages aux États-Unis) [2], ce qui représente une multiplication de 2,5 du nombre de voyages à l'étranger. En comparaison, la population canadienne ne s'est accrue que de $13 \%$ au cours de la même période, ce qui laisse croire que cette augmentation des voyages n'est pas uniquement liée à la croissance de la population (3). 
La hausse de l'incidence de la rougeole dans les pays où les échanges de population avec le Canada sont élevés est un autre facteur contributif pouvant expliquer l'augmentation du nombre de cas importés. Par exemple, d'importantes éclosions de rougeole en France (2011) [4], aux Pays-Bas (2013) [5] et aux Philippines (2014) [6] peuvent avoir participé à l'augmentation de l'importation de cas de rougeole au Canada.

En 2014, la propagation secondaire de la plupart des cas importés a été restreinte. Sur les 21 cas importés rapporté au 10 mai, huits ont entraîné une transmission au Canada. Sur les cinq cas qui avaient connu une issue au moment de la rédaction, le délai médian qui séparait la date d'apparition de l'éruption cutanée du premier et du dernier cas était de 20 jours (intervalle : de 13 à 35) et le nombre médian de cas était de 3 (intervalle : de 2 à 10). Parmi les cas importés, la majorité n'avait pas été vaccinée $(n=12 ; 57 \%)$ ou présentait des antécédents vaccinaux inconnus $(n=4 ; 19 \%)$ et le reste avait reçu une $(n=1 ; 5 \%)$ ou deux doses $(n=4$; $19 \%)$ du vaccin contenant le virus de la rougeole. La Figure 1 illustre la distribution en fonction de l'âge des cas de rougeole, selon la source d'exposition.

Figure 1. Distribution en fonction de l'âge des cas confirmés de rougeole au Canada, du 30 décembre 2013 au 10 mai 2014 ( $n=103$ )

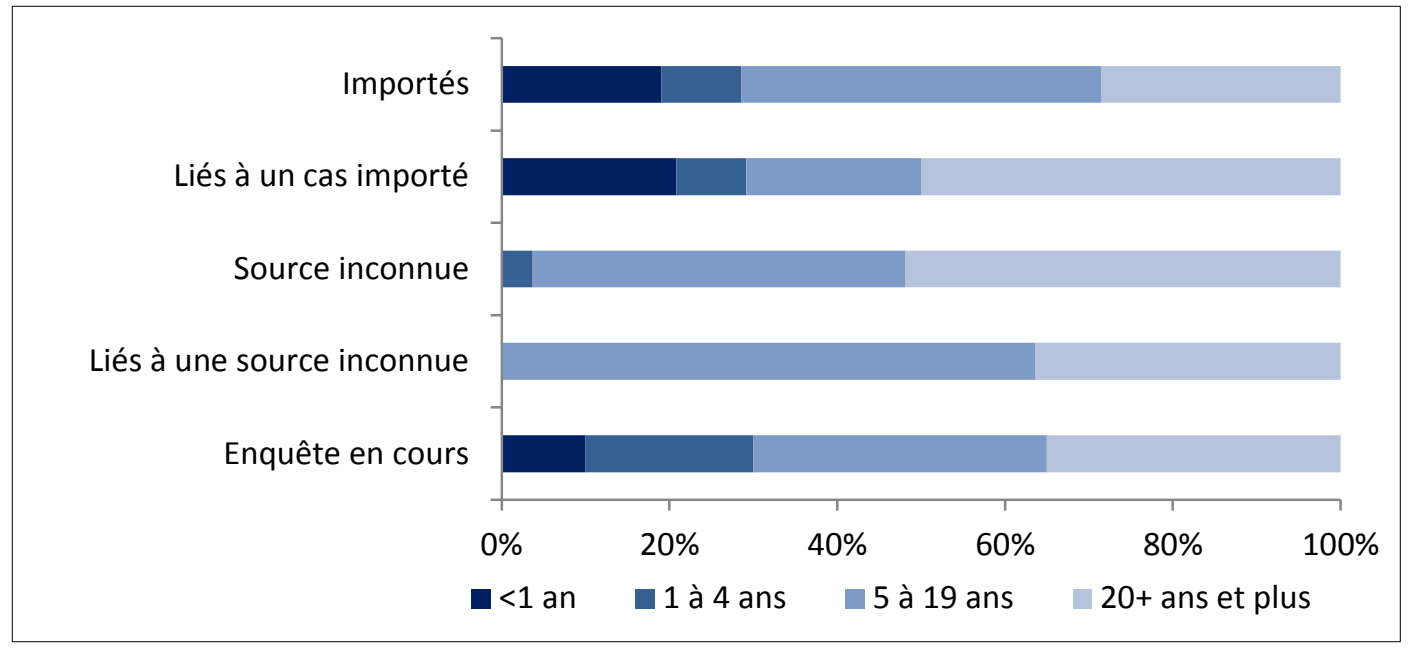

\section{Limites}

Les renseignements fournis dans le présent communiqué sont limités à ce qui a été signalé à l'Agence par l'entremise du Système canadien de surveillance de la rougeole et de la rubéole ou du projet pilote du Système de surveillance de la rougeole et de la rubéole au 10 mai 2014. Par conséquent, il peut y avoir un manque d'uniformité par rapport aux données signalées par les provinces et les territoires. Une vérification des données est effectuée annuellement auprès des provinces et des territoires afin de valider les données sur la rougeole signalées à l'Agence.

\section{Plan d'action du Canada pour le maintien de l'élimination de la rougeole}

Le Canada reconnaît que la menace d'importation de cas de rougeole, conjuguée à une couverture vaccinale sous-optimale dans certaines régions, pose un risque de réintroduction et de transmission au pays, comme on a pu l'observer lors d'éclosions de 2011 de rougeole qui ont mis en péril l'élimination de la maladie au Canada. Afin d'éliminer la rougeole de manière durable, il faut nécessairement renforcer les programmes d'immunisation, continuer à assurer une vigilance accrue et endiguer rapidement les cas d'infection importés.

Pour que l'élimination de la rougeole et de la rubéole soit maintenue au Canada, tous les ordres de gouvernement devront continuer à renforcer leur collaboration et à appuyer des programmes d'immunisation de haute qualité. L'Agence prévoit consulter ses partenaires provinciaux et territoriaux, ainsi que d'autres experts à 
l'échelle nationale et internationale afin de dresser et de mettre en œuvre un plan d'action pluriannuel tirant parti des réalisations antérieures pour s'assurer que l'élimination de la rougeole est maintenue.

\section{Références}

(1) King A, Varughese P, De Serres G, Tipples GA, Waters J, Working Group on Measles Elimination. Measles elimination in Canada. J Infect Dis. 2004;189 Suppl 1:S236-42. doi: 10.1086/378499.

(2) Statistique Canada. Enquête sur les voyages internationaux (résidents canadiens 2008-2012). Extrait personnalisé pour l'Agence de la santé publique du Canada. 2014.

(3) Statistique Canada, Division de la démographie, Section des estimations démographiques. Estimations de la population pour juillet, 2011. Estimations intercensitaires finales.

(4) Organisation mondiale de la Santé (région de l'Europe). Système d'information sur les maladies infectieuses. http://data.euro.who.int/cisid/?TabID=67.

(5) Knol MJ, Urbanus AT, Swart EM, et al. Large ongoing measles outbreak in a religious community in the Netherlands since May 2013. Eurosurveillance. 2013; 18(36).

(6) World Health Organization Western Pacific Region. Measles-rubella bulletin (April 2014). http://www.wpro.who.int/immunization/documents/MRBulletinVol8Issue04.pdf?ua=1. 